\title{
Very low food security predicts obesity predominantly in California Hispanic men and women
}

\author{
Cindy W Leung ${ }^{1,2, *}$, David R Williams ${ }^{3}$ and Eduardo Villamor ${ }^{1,2,4}$ \\ 'Department of Nutrition, Harvard School of Public Health, Boston, MA, USA: ${ }^{2}$ Department of Epidemiology, \\ Harvard School of Public Health, 665 Huntington Avenue, Boston, MA 021 15, USA: ${ }^{3}$ Department of Society, \\ Human Development and Health, Harvard School of Public Health, Boston, MA, USA: ${ }^{4}$ Department of \\ Epidemiology, School of Public Health, University of Michigan, Ann Arbor, MI, USA
}

Submitted 7 September 2011: Final revision received 31 January 2012: Accepted 13 February 2012: First published online 2 April 2012

\begin{abstract}
Objective: A high prevalence of food insecurity has persisted in the USA for the past two decades. Previous studies suggest that the association between food insecurity and obesity may vary by gender and race/ethnicity. We examined whether food insecurity was associated with BMI and obesity within gender and racial/ethnic groups in a large, diverse sample of low-income adults.

Design: A cross-sectional analysis of a large population-based health survey. We compared the distribution of BMI and obesity by food security levels within gender and racial/ethnic categories.

Setting: Data were derived from the 2003-2009 waves of the California Health Interview Survey.

Subjects: The study sample included 35747 non-elderly adults with households $\leq 200 \%$ of the federal poverty level.

Results: Among Hispanic men, very low food security was associated with a $1 \cdot 0 \mathrm{~kg} / \mathrm{m}^{2}$ higher BMI $\left(95 \%\right.$ CI $\left.0 \cdot 3,1 \cdot 7 \mathrm{~kg} / \mathrm{m}^{2}\right)$ and a $36 \%$ higher prevalence of obesity (95\% CI 17, 58\%) after multivariate adjustment. Among Hispanic women, very low food security was associated with a $1 \cdot 1 \mathrm{~kg} / \mathrm{m}^{2}$ higher BMI $(95 \% \mathrm{CI} 0 \cdot 4$, $1.9 \mathrm{~kg} / \mathrm{m}^{2}$ ) and a $22 \%$ higher prevalence of obesity (95\% CI 8, 38\%). Positive associations were also observed for Asian women and multi-racial men. No significant associations were observed for non-Hispanic whites, African Americans, Asian men or multi-racial women.

Conclusions: Our results suggest that the association of food insecurity and obesity is limited to individuals of certain low-income, minority racial/ethnic groups. Whether targeted interventions to address food insecurity in these individuals may also decrease obesity risk deserves further investigation.
\end{abstract}

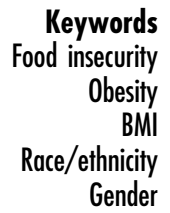

It has been well established that prevalence rates of obesity and obesity-related health conditions have grown considerably in the USA over the past several decades. Obesity disproportionately affects minority and lowincome populations, especially black women, Hispanic women, Native Americans, Pacific Islanders and men and women of low socio-economic status ${ }^{(1)}$, with prevalences as high as $40-50 \%$ in some of these groups ${ }^{(2)}$.

Although not as dramatic as the trend in obesity, the prevalence of food insecurity, defined as the limited or uncertain availability of nutritionally adequate and safe foods or the limited or uncertain ability to acquire acceptable foods in socially acceptable ways ${ }^{\text {,(3) }}$, has also persisted at high levels for the past two decades. In 2007 , it was estimated that $11 \cdot 1 \%$ of all US households were food insecure; this proportion has since increased to $14.7 \%{ }^{(4)}$. As with obesity, there is also considerable variation in the prevalence of food insecurity. Single women with children, black households, Hispanic households and households living under the federal poverty level (FPL) all have higher prevalences of food insecurity compared with the national average ${ }^{(5)}$.

Food insecurity has been associated with poorer health profiles $^{(6)}$, including overweight and obesity ${ }^{(7-14)}$ and metabolic syndrome ${ }^{(15)}$, among low-income adults and particularly low-income women. In one of the earlier studies, Townsend and colleagues observed that women with mild food insecurity were $30 \%$ more likely to be overweight (BMI $>27 \cdot 3 \mathrm{~kg} / \mathrm{m}^{2}$ ) than food-secure women; the same association was not observed among men ${ }^{(11)}$. Adams and colleagues noted that after adjustment for income and education, the associations between food 
insecurity and obesity were stronger among Asian, black and Hispanic women collectively, than among white women, in the 1998-99 California Women's Health Survey ${ }^{(9)}$. In another study, Kaiser and colleagues found that food-insecure Hispanic/Latino women were 98\% more likely to be obese (BMI $>30 \cdot 0 \mathrm{~kg} / \mathrm{m}^{2}$ ) than their food-secure counterparts in a convenience sample of Hispanic/Latino women ${ }^{(10)}$. These studies suggest that the association between food insecurity and obesity may vary by gender and race/ethnicity; however, few studies have been able to examine the degree to which this association is modified by gender and race/ethnicity. Depending on socio-economic influences, acculturation status or other social factors, some groups, such as white adults or men, may adopt better coping strategies for food insecurity than black or Hispanic adults or women.

The objective of the present study was to examine the heterogeneity in the associations of food insecurity with BMI and obesity within different gender and racial/ethnic groups, using a large, diverse sample of low-income California adults. Based on existing evidence, we hypothesized that food insecurity would be more strongly associated with BMI or obesity among African American and Hispanic women, when compared with other groups.

\section{Methods}

\section{Study population}

The California Health Interview Survey (CHIS) is a large, population-based, random-digit-dial telephone survey of households, aimed to be representative of California's non-institutionalized population ${ }^{(16)}$. CHIS has been conducted every two years since 2001 and is the largest statewide health survey, collecting information on a range of health behaviours and health outcomes. CHIS provides a unique opportunity for studying health disparities due to the oversampling of California's large population of minority racial/ethnic groups, particularly Hispanics and Asians $^{(17)}$. Interviews are conducted in English, Spanish, Chinese (Mandarin and Cantonese dialects), Vietnamese and Korean ${ }^{(18)}$.

For the present study, data were combined from the 2003, 2005, 2007 and 2009 CHIS, to ensure a sufficient representation of individuals from minority racial/ethnic groups. The analytical sample was restricted to households whose incomes fell at or below $200 \%$ of the FPL, in order to exclude higher-income households who were unlikely to experience food insecurity ${ }^{(19,20)}$ and to provide a useful comparison of food insecurity among households of similar socio-economic status. In 2009, $200 \%$ of the FPL translated into a threshold of \$US 44100 per annum for a family of four ${ }^{(21)}$. We further restricted our sample to adults aged 18-65 years and women who were not pregnant at the time of the survey to avoid potential misclassification of the outcome measures. Response rates from the 2003-2007 surveys ranged from $18 \cdot 7 \%$ to $33 \cdot 5 \%^{(22-24)}$. The 2009 survey response rate has not yet been published.

\section{Assessment of bousehold food security status}

Food insecurity was assessed over the past 12 months and was measured using the six-item short form of the US Household Food Security Survey Module ${ }^{(25)}$. Questions are ordered by range of severity of food insecurity and attribute related experiences or behaviours to insufficient resources to buy food. Responses of 'sometimes true', 'often true', 'almost every month', 'some months but not every month' and 'yes' were coded as affirmative. A raw score ranging from 0 to 6 was created by summing the affirmatives of the questions. Food insecurity was classified according to the US Department of Agriculture (USDA) guidelines: $0-1$, high or marginal food security; 2-4, low food security; 5-6, very low food security. It should be noted that we use 'food insecurity' as a broad term that encompasses households reporting either low food security or very low food security. We present results by low food security or very low food security status, to maintain consistency with the USDA classifications.

\section{Outcomes}

The outcomes for analyses were BMI and obesity. BMI was calculated from self-reported height and weight data as $\mathrm{kg} / \mathrm{m}^{2}$. Obesity was defined as BMI $\geq 30 \cdot 0 \mathrm{~kg} / \mathrm{m}^{2}$.

\section{Covariates}

Covariates for multivariate regression analyses included age (18-30, 31-40, 41-50, 51-65 years), gender, race/ethnicity, birthplace (US-born; born outside USA), English proficiency (speaks only English; speaks English well or very well; speaks English not well or not at all), employment status at time of survey (employed; unemployed), poverty level (0-24.9\%, 25-49.9\%, 50-74.9\%, 75-99.9\%, 100-124.9\%, 125-149.9\%, 150-174.9\%, 175-200\% FPL), education (high school diploma/fewer than 12 years of education; any college or higher), self-reported health status (excellentgood; fair-poor), smoking status (never smoker; former smoker; current smoker) and family type (single or married without children; married with children; single with children). For each of the categorical variables, the first group was used as the reference category.

As CHIS had multiple definitions for race/ethnicity, we used the definitions from the Office of Management and Budget: Hispanic, non-Hispanic (NH) white, NH African American, NH American Indian/Alaskan Native (AIAN), NH Asian, NH Native Hawaiian/Pacific Islander (NHPI), $\mathrm{NH}$ other singular race, and $\mathrm{NH}$ two or more races. Due to small sample sizes, we excluded individuals of AIAN descent ( $n$ 461), NHPI descent ( $n$ 87) or other singular race $(n 65)$. The final analytical sample comprised 35747 adults. 


\section{Statistical analysis}

Statistical analyses were performed using the Stata/IC for Windows statistical software package version $11 \cdot 1$ (StataCorp LP, College Station, TX, USA). Using Stata's survey data estimation procedures, complex survey weights were used in all analyses to account for the different sampling probabilities and potential non-response bias, and to obtain effect estimates and standard errors representative of the California adult population. Variances were calculated using replicate weights provided with the public-use data files. All replicate weights were recalculated to reflect the probability of being sampled in the pooled survey years according to CHIS guidelines.

First, we compared the distribution of food security status according to sociodemographic characteristics using $\chi^{2}$ tests. Next, we used multivariate linear regression models to examine the relationships between low food security and very low food security with continuous BMI. We also estimated prevalence ratios and 95\% confidence intervals for obesity by categories of food security using multivariate Poisson regression models. Because the aim of the study was to examine the heterogeneity of the associations between food insecurity, BMI and obesity, we tested two-way cross-product terms between gender and race/ethnicity with low food security or very low food security in regression models. We also tested three-way interactions between gender, race/ethnicity and low food security or very low food security. Last, we examined whether the associations between low food security and very low food security with BMI or obesity within racial/ethnic groups were further modified by birthplace or indicators of economic adversity (poverty level, education, employment status at time of survey).

We examined the appropriateness of combining data from surveys conducted in four different years by computing two-way interaction terms between food insecurity and survey year in the models for BMI and obesity. We also estimated the prevalences of high/marginal, low and very low food security for the entire CHIS sample and the analytical sample by year of survey and compared these with national estimates from the USDA Economic Research
Service $^{(26)}$. All statistical tests were two-sided and significance was considered at $P<0 \cdot 05$.

\section{Results}

Between 2003 and 2009, prevalences of low food security and very low food security in the entire CHIS sample ranged from $6 \cdot 5 \%$ to $9 \cdot 0 \%$ and from $2 \cdot 9 \%$ to $5 \cdot 3 \%$, respectively (Table 1 ). Aside from a slight decline in the prevalence of very low food security in the 2005 CHIS, these figures were comparable to the national estimates for the same years, as reported by the USDA ${ }^{(3,4,27,28)}$. Both estimates from CHIS and from the USDA were reported at the household level. In the low-income CHIS sample of 35747 adults, the prevalence of low food security ranged from $21 \cdot 8 \%$ to $26 \cdot 8 \%$ throughout the study period, whereas the prevalence of very low food security ranged from $10 \cdot 2 \%$ to $16 \cdot 2 \%$. Adults were most likely to report experiencing low or very low food security in the past 12 months if they were aged 31-50 years, of female gender, of African American or Hispanic descent, had the lowest English proficiency, were living under the FPL, had 12 or fewer years of education, reported poorer general health status or were single parents (Table 2).

Among all adults, very low food security was associated with a $0.7 \mathrm{~kg} / \mathrm{m}^{2}$ higher adjusted BMI (95\% CI $0 \cdot 4$, $\left.1 \cdot 1 \mathrm{~kg} / \mathrm{m}^{2}, P<0 \cdot 001\right)$ and a $16 \%$ higher adjusted prevalence of obesity (prevalence ratio $(P R)=1 \cdot 16$, 95\% CI $1 \cdot 07,1 \cdot 26, P=0 \cdot 001$; results not shown). However, there was evidence of heterogeneity in the associations of food security status with BMI $\left(P_{\text {interaction }}=0 \cdot 02\right)$ by race/ ethnicity and gender. Among Hispanic men, having very low food security was associated with a $1.0 \mathrm{~kg} / \mathrm{m}^{2}$ higher BMI $(P=0.003)$ and a $36 \%$ higher prevalence of obesity $(P<0 \cdot 001)$ when compared with food-secure Hispanic men (Table 3). Similarly, for multi-racial men, very low food security was significantly associated with a $1.9 \mathrm{~kg} / \mathrm{m}^{2}$ higher BMI $(P=0.03)$ and a $16 \%$ higher prevalence of obesity $(P=0 \cdot 001)$. No associations were observed for $\mathrm{NH}$ white, African American or Asian men.

Table 1 Food security status in the USA and the California Health Interview Survey (CHIS), 2003-2009

\begin{tabular}{|c|c|c|c|c|}
\hline & \multicolumn{4}{|c|}{ CHIS survey year } \\
\hline & 2003 & 2005 & 2007 & 2009 \\
\hline \multicolumn{5}{|c|}{ Food security status in the US population* } \\
\hline Low food security (\%) & $7 \cdot 7$ & $7 \cdot 1$ & $7 \cdot 0$ & $9 \cdot 0$ \\
\hline Very low food security (\%) & 3.5 & $3 \cdot 9$ & $4 \cdot 1$ & $5 \cdot 7$ \\
\hline \multicolumn{5}{|c|}{ Food security status in the entire CHIS sample } \\
\hline Low food security (\%) & $8 \cdot 0$ & $6 \cdot 5$ & $7 \cdot 1$ & $9 \cdot 0$ \\
\hline Very low food security & 3.5 & $2 \cdot 9$ & $3 \cdot 6$ & $5 \cdot 3$ \\
\hline \multicolumn{5}{|c|}{ Food security status in the low-income CHIS samplet } \\
\hline Low food security (\%) & $24 \cdot 9$ & $21 \cdot 8$ & $24 \cdot 6$ & $26 \cdot 8$ \\
\hline Very low food security (\%) & $11 \cdot 1$ & $10 \cdot 2$ & $13 \cdot 0$ & $16 \cdot 2$ \\
\hline
\end{tabular}

*Data from the US Department of Agriculture, Economic Research Service.

tAdults with household incomes $\leq 200 \%$ of the federal poverty level, aged 18-65 years, not pregnant at the time of the survey. 
Table 2 Sociodemographic characteristics and distribution of food security status among low-income adults: California Health Interview Survey, 2003-2009*

\begin{tabular}{|c|c|c|c|c|c|c|}
\hline & \multirow[b]{2}{*}{ Raw $n$} & \multirow[b]{2}{*}{ Weighted \% } & \multicolumn{2}{|c|}{ Low food security } & \multicolumn{2}{|c|}{ Very low food security } \\
\hline & & & $\%$ & SE & $\%$ & SE \\
\hline \multicolumn{7}{|l|}{ Age } \\
\hline $18-30$ years & 9155 & $36 \cdot 5$ & $20 \cdot 2$ & 0.7 & $9 \cdot 8$ & 0.5 \\
\hline $31-40$ years & 7862 & $24 \cdot 6$ & $28 \cdot \overline{9}$ & 0.8 & $12 \cdot 3$ & 0.6 \\
\hline $41-50$ years & 8130 & $21 \cdot 1$ & $27 \cdot 7$ & $1 \cdot 1$ & $16 \cdot 9$ & $1 \cdot 1$ \\
\hline $51-65$ years & 10600 & $17 \cdot 9$ & $24 \cdot 0$ & 0.8 & $14 \cdot 5$ & 0.6 \\
\hline \multicolumn{7}{|l|}{ Sex } \\
\hline Male & 13643 & $47 \cdot 6$ & $24 \cdot 1$ & 0.6 & $12 \cdot 1$ & 0.5 \\
\hline Female & 22104 & $52 \cdot 4$ & $25 \cdot 1$ & 0.5 & $13 \cdot 4$ & 0.4 \\
\hline \multicolumn{7}{|l|}{ Race/ethnicity } \\
\hline Non-Hispanic white & 12562 & $21 \cdot 4$ & $18 \cdot 7$ & 0.7 & $16 \cdot 6$ & 0.8 \\
\hline African American & 2218 & $6 \cdot 8$ & $21 \cdot 8$ & $1 \cdot 4$ & $20 \cdot 2$ & $1 \cdot 7$ \\
\hline Hispanic/Latino & 16100 & $60 \cdot 0$ & $27 \cdot 4$ & 0.6 & $11 \cdot \overline{8}$ & 0.4 \\
\hline Asian & 3753 & $10 \cdot 5$ & $22 \cdot 9$ & $1 \cdot 5$ & $4 \cdot 9$ & $0 \cdot 8$ \\
\hline Multi-racial & 1114 & $1 \cdot 4$ & $20 \cdot 1$ & 1.9 & $19 \cdot 4$ & 1.9 \\
\hline \multicolumn{7}{|l|}{ Birthplace } \\
\hline US-born & 19563 & $44 \cdot 1$ & $18 \cdot 8$ & 0.5 & $15 \cdot 4$ & 0.6 \\
\hline Born outside USA & 16184 & $55 \cdot 9$ & $29 \cdot 2$ & 0.6 & $10 \cdot 6$ & 0.4 \\
\hline \multicolumn{7}{|l|}{ English proficiency } \\
\hline Speaks only English & 15965 & $32 \cdot 0$ & $19 \cdot 2$ & 0.6 & $16 \cdot 9$ & 0.7 \\
\hline Speaks English well or very well & 8853 & $29 \cdot 2$ & $19 \cdot 7$ & 0.7 & $9 \cdot 5$ & 0.6 \\
\hline Speaks English not well or not at all & 10929 & $38 \cdot 9$ & $32 \cdot 8$ & 0.8 & $11 \cdot 8$ & 0.5 \\
\hline \multicolumn{7}{|l|}{ Employment status } \\
\hline Employed & 20306 & $62 \cdot 9$ & $25 \cdot 5$ & 0.6 & $10 \cdot 4$ & 0.3 \\
\hline Unemployed & 15441 & $37 \cdot 1$ & $23 \cdot 1$ & 0.7 & $16 \cdot 8$ & 0.7 \\
\hline \multicolumn{7}{|l|}{ Poverty level } \\
\hline $0-24.9 \% \mathrm{FPL}$ & 2750 & $7 \cdot 7$ & $24 \cdot 6$ & $1 \cdot 3$ & $16 \cdot 4$ & $1 \cdot 5$ \\
\hline $25-49.9 \% \mathrm{FPL}$ & 2301 & $7 \cdot 2$ & $32 \cdot 5$ & 1.9 & $16 \cdot 4$ & $1 \cdot 4$ \\
\hline $50-74.9 \% \mathrm{FPL}$ & 4270 & $13 \cdot \overline{7}$ & $29 \cdot 2$ & $1 \cdot 4$ & $16 \cdot 2$ & $1 \cdot 4$ \\
\hline $75-99.9 \% \mathrm{FPL}$ & 6308 & $17 \cdot 5$ & $26 \cdot \overline{5}$ & $1 \cdot 0$ & $14 \cdot \overline{5}$ & 0.7 \\
\hline $100-124 \cdot 9 \%$ FPL & 5516 & $14 \cdot 8$ & $25 \cdot 6$ & $1 \cdot 1$ & $13 \cdot 0$ & $0 \cdot 8$ \\
\hline $125-149.9 \% \mathrm{FPL}$ & 5032 & $13 \cdot 9$ & $22 \cdot 7$ & $1 \cdot 1$ & $10 \cdot 4$ & 0.8 \\
\hline $150-174.9 \% \mathrm{FPL}$ & 4788 & $12 \cdot 7$ & $20 \cdot 7$ & $1 \cdot 1$ & $9 \cdot 2$ & 0.9 \\
\hline $175-200 \% \mathrm{FPL}$ & 4782 & $12 \cdot 4$ & $17 \cdot 2$ & 0.9 & $8 \cdot 0$ & 0.7 \\
\hline \multicolumn{7}{|l|}{ Education } \\
\hline Any college or higher & 14167 & $30 \cdot 4$ & $18 \cdot 9$ & 0.6 & $12 \cdot 4$ & 0.6 \\
\hline High school diploma/fewer than 12 years of education & 21580 & $69 \cdot 6$ & $27 \cdot 1$ & 0.5 & $12 \cdot 9$ & 0.4 \\
\hline \multicolumn{7}{|l|}{ General health status } \\
\hline Excellent-good & 23442 & $67 \cdot 8$ & $21 \cdot 4$ & 0.5 & $10 \cdot 0$ & 0.4 \\
\hline Fair-poor & 12305 & $32 \cdot 3$ & $31 \cdot 4$ & 0.8 & $18 \cdot 6$ & 0.6 \\
\hline \multicolumn{7}{|l|}{ Smoking status } \\
\hline Never smoker & 21064 & $64 \cdot 0$ & $24 \cdot 6$ & 0.6 & $10 \cdot 6$ & 0.4 \\
\hline Former smoker & 6918 & $16 \cdot 4$ & $24 \cdot 7$ & $1 \cdot 0$ & $13 \cdot 0$ & 0.8 \\
\hline Current smoker & 7765 & $19 \cdot 6$ & $24 \cdot 5$ & 0.9 & $19 \cdot 6$ & 0.8 \\
\hline \multicolumn{7}{|l|}{ Family type } \\
\hline Single or married without children & 18878 & $50 \cdot 6$ & $21 \cdot 5$ & 0.5 & $13 \cdot 4$ & 0.6 \\
\hline Married with children & 11318 & $37 \cdot 3$ & $27 \cdot 7$ & 0.7 & $10 \cdot 4$ & 0.4 \\
\hline Single with children & 5551 & $12 \cdot 2$ & $27 \cdot 9$ & $1 \cdot 2$ & $17 \cdot 5$ & 0.9 \\
\hline
\end{tabular}

FPL, federal poverty level.

${ }^{*}$ All differences in sociodemographic characteristics by food security status were statistically significant at $P<0 \cdot 05$, as assessed by $\chi^{2}$ tests.

Hispanic women with very low food security had a $1 \cdot 1 \mathrm{~kg} / \mathrm{m}^{2}$ higher BMI $(P=0 \cdot 003)$ and a $22 \%$ higher prevalence of obesity $(P=0 \cdot 001)$, compared with foodsecure Hispanic women (Table 4). For Asian women, low food security was associated with a $1 \cdot 1 \mathrm{~kg} / \mathrm{m}^{2}$ significantly higher BMI $(P=0 \cdot 008)$ compared with foodsecure Asian women. No associations were observed for NH white, African American or multi-racial women. There was also no evidence of further effect modification by birthplace or indicators of economic adversity on any of the associations examined $(P>0 \cdot 1)$.

\section{Discussion}

Our results provide evidence that experiencing very low food security is related to higher BMI and higher prevalence of obesity in low-income Hispanic men, multiracial men and Hispanic women. Low food security was also related to higher BMI for Asian women. No significant associations were observed for $\mathrm{NH}$ white men and women, African American men and women, Asian men or multi-racial women. There was also no further modification of these associations by a participant's 
Table 3 Associations of food security status with BMI and obesity among low-income men: California Health Interview Survey, 2003-2009

\begin{tabular}{|c|c|c|c|c|c|c|c|c|c|c|}
\hline & \multirow[b]{3}{*}{$n$} & \multirow[b]{3}{*}{$\%$} & \multirow{2}{*}{\multicolumn{4}{|c|}{ BMI $\left(\mathrm{kg} / \mathrm{m}^{2}\right)$}} & \multicolumn{4}{|c|}{ Obesity $\left(\mathrm{BMI} \geq 30 \mathrm{~kg} / \mathrm{m}^{2}\right)$} \\
\hline & & & & & & & \multicolumn{2}{|c|}{ Weighted \% } & \multirow[b]{2}{*}{ Adjusted PR* } & \multirow[b]{2}{*}{$95 \% \mathrm{Cl}$} \\
\hline & & & Mean & $\mathrm{SE}$ & Adjusted difference* & $95 \% \mathrm{Cl}$ & Mean & SE & & \\
\hline \multicolumn{11}{|l|}{ All low-income men } \\
\hline High/marginal food security & 9014 & 64 & $27 \cdot 3$ & $0 \cdot 1$ & Ref. & & $24 \cdot 5$ & $0 \cdot 1$ & Ref. & \\
\hline Low food security & 2996 & 24 & $27 \cdot 7$ & $0 \cdot 2$ & 0.0 & $-0.3,0.4$ & $24 \cdot 6$ & $1 \cdot 3$ & 0.98 & $0.87,1.09$ \\
\hline Very low food security & 1627 & 12 & $28 \cdot 4$ & $0 \cdot 2$ & $0 \cdot 6$ & $0 \cdot 1,1 \cdot 0$ & $33 \cdot 4$ & $2 \cdot 1$ & $1 \cdot 19$ & $1 \cdot 04,1 \cdot 37$ \\
\hline \multicolumn{11}{|l|}{ Non-Hispanic white } \\
\hline High/marginal food security & 3290 & 68 & $27 \cdot 0$ & $0 \cdot 2$ & Ref. & & $23 \cdot 5$ & $1 \cdot 3$ & Ref. & \\
\hline Low food security & 815 & 18 & $27 \cdot 0$ & $0 \cdot 4$ & $-0 \cdot 0$ & $-0 \cdot 8,0 \cdot 7$ & $25 \cdot 5$ & $3 \cdot 0$ & 1.04 & $0 \cdot 83,1 \cdot 31$ \\
\hline Very low food security & 642 & 14 & $27 \cdot 2$ & $0 \cdot 3$ & -0.2 & $-0.9,0.4$ & $25 \cdot 2$ & $2 \cdot 9$ & 0.95 & $0.73,1.23$ \\
\hline \multicolumn{11}{|l|}{ African American } \\
\hline High/marginal food security & 409 & 59 & $27 \cdot 4$ & 0.5 & Ref. & & $28 \cdot 7$ & $3 \cdot 8$ & Ref. & \\
\hline Low food security & 141 & 20 & $27 \cdot 7$ & $0 \cdot 7$ & $0 \cdot 1$ & $-1 \cdot 4,1 \cdot 5$ & $31 \cdot 6$ & $6 \cdot 1$ & 1.06 & $0.70,1.59$ \\
\hline Very low food security & 150 & 22 & $27 \cdot 3$ & $0 \cdot 6$ & -0.5 & $-1.9,0.9$ & $24 \cdot 4$ & $5 \cdot 2$ & 0.76 & $0.48,1.22$ \\
\hline \multicolumn{11}{|l|}{ Hispanic/Latino } \\
\hline High/marginal food security & 3901 & 62 & $28 \cdot 0$ & $0 \cdot 1$ & Ref. & & $27 \cdot 3$ & $1 \cdot 0$ & Ref. & \\
\hline Low food security & 1642 & 27 & $28 \cdot 2$ & $0 \cdot 2$ & $0 \cdot 0$ & $-0.4,0.5$ & $25 \cdot 8$ & $1 \cdot 6$ & 0.95 & $0.83,1 \cdot 10$ \\
\hline Very low food security & 687 & 11 & $29 \cdot 4$ & $0 \cdot 3$ & $1 \cdot 0$ & $0 \cdot 3,1 \cdot 7$ & $40 \cdot 6$ & $2 \cdot 8$ & $1 \cdot 36$ & $1 \cdot 17,1.58$ \\
\hline \multicolumn{11}{|l|}{ Asian } \\
\hline High/marginal food security & 1193 & 72 & $24 \cdot 8$ & $0 \cdot 3$ & Ref. & & $10 \cdot 5$ & $1 \cdot 6$ & Ref. & \\
\hline Low food security & 305 & 23 & $25 \cdot 0$ & 0.5 & $0 \cdot 2$ & $-0 \cdot 8,1 \cdot 1$ & $12 \cdot 0$ & $3 \cdot 2$ & $1 \cdot 31$ & $0 \cdot 76,2 \cdot 25$ \\
\hline Very low food security & 76 & 6 & $25 \cdot 6$ & $0 \cdot 8$ & $0 \cdot 1$ & $-1 \cdot 8,2 \cdot 0$ & $9 \cdot 4$ & $4 \cdot 2$ & 0.97 & $0.35,2.69$ \\
\hline \multicolumn{11}{|l|}{ Multi-racial } \\
\hline High/marginal food security & 221 & 61 & $25 \cdot 7$ & 0.5 & Ref. & & $14 \cdot 0$ & $3 \cdot 8$ & & \\
\hline Low food security & 93 & 18 & $26 \cdot 8$ & $1 \cdot 0$ & $1 \cdot 3$ & $-0.3,2.9$ & $17 \cdot 5$ & $4 \cdot 7$ & 1.02 & $0.95,1.08$ \\
\hline Very low food security & 72 & 21 & $28 \cdot 3$ & $1 \cdot 1$ & $1 \cdot 9$ & $0 \cdot 2,3 \cdot 5$ & $28 \cdot 6$ & $9 \cdot 5$ & $1 \cdot 16$ & $1 \cdot 07,1 \cdot 26$ \\
\hline
\end{tabular}

Ref., reference category.

${ }^{*}$ From linear and Poisson regression models adjusted for age categories, birthplace, English proficiency, general health status, smoking status, family type, employment status, poverty level and education level. Models for all low-income men additionally adjusted for race/ethnicity.

Table 4 Associations of food security status with BMI and obesity among low-income women: California Health Interview Survey, 2003-2009

\begin{tabular}{|c|c|c|c|c|c|c|c|c|c|c|}
\hline & \multirow[b]{3}{*}{$n$} & \multirow[b]{3}{*}{$\%$} & \multirow{2}{*}{\multicolumn{4}{|c|}{ BMI $\left(\mathrm{kg} / \mathrm{m}^{2}\right)$}} & \multicolumn{4}{|c|}{ Obesity $\left(\mathrm{BMI} \geq 30 \mathrm{~kg} / \mathrm{m}^{2}\right)$} \\
\hline & & & & & & & \multicolumn{2}{|c|}{ Weighted \% } & \multirow[b]{2}{*}{ Adjusted PR* } & \multirow[b]{2}{*}{$95 \% \mathrm{Cl}$} \\
\hline & & & Mean & SE & Adjusted difference* & $95 \% \mathrm{Cl}$ & Mean & SE & & \\
\hline \multicolumn{11}{|l|}{ All low-income women } \\
\hline High/marginal food security & 13482 & 62 & $26 \cdot 9$ & $0 \cdot 1$ & Ref. & & $24 \cdot 8$ & 0.6 & Ref. & \\
\hline Low food security & 5342 & 25 & $28 \cdot 1$ & $0 \cdot 2$ & $0 \cdot 3$ & $-0 \cdot 0,0 \cdot 7$ & $29 \cdot 7$ & $1 \cdot 0$ & $1 \cdot 04$ & $0.97,1.13$ \\
\hline Very low food security & 3273 & 13 & $29 \cdot 0$ & 0.3 & 0.9 & $0 \cdot 4,1 \cdot 4$ & $35 \cdot 1$ & 1.6 & $1 \cdot 14$ & $1 \cdot 04,1 \cdot 25$ \\
\hline \multicolumn{11}{|l|}{ Non-Hispanic white } \\
\hline High/marginal food security & 4883 & 63 & $26 \cdot 3$ & $0 \cdot 1$ & Ref. & & $23 \cdot 7$ & 0.9 & Ref. & \\
\hline Low food security & 1515 & 19 & $27 \cdot 3$ & $0 \cdot 3$ & 0.6 & $-0 \cdot 1,1 \cdot 2$ & $28 \cdot 6$ & $2 \cdot 0$ & $1 \cdot 09$ & $0.95,1.25$ \\
\hline Very low food security & 1409 & 19 & $27 \cdot 6$ & $0 \cdot 4$ & $0 \cdot 3$ & $-0 \cdot 3,1 \cdot 0$ & $28 \cdot 5$ & $2 \cdot 6$ & $1 \cdot 01$ & $0.85,1 \cdot 21$ \\
\hline \multicolumn{11}{|l|}{ African American } \\
\hline High/marginal food security & 860 & 57 & $28 \cdot 5$ & $0 \cdot 4$ & Ref. & & $36 \cdot 5$ & $2 \cdot 6$ & Ref. & \\
\hline Low food security & 359 & 24 & $29 \cdot 9$ & $0 \cdot 6$ & $0 \cdot 4$ & $-0 \cdot 9,1 \cdot 8$ & $40 \cdot 1$ & $3 \cdot 8$ & $1 \cdot 06$ & $0 \cdot 85,1 \cdot 31$ \\
\hline Very low food security & 299 & 19 & $30 \cdot 3$ & $1 \cdot 1$ & $1 \cdot 4$ & $-0 \cdot 4,3 \cdot 2$ & $37 \cdot 2$ & $5 \cdot 3$ & $1 \cdot 00$ & $0.75,1.32$ \\
\hline \multicolumn{11}{|l|}{ Hispanic/Latino } \\
\hline High/marginal food security & 5763 & 60 & $27 \cdot 8$ & $0 \cdot 1$ & Ref. & & $28 \cdot 5$ & 0.9 & Ref. & \\
\hline Low food security & 2838 & 28 & $28 \cdot 6$ & $0 \cdot 2$ & $0 \cdot 2$ & $-0.3,0.6$ & $31 \cdot 7$ & $1 \cdot 3$ & $1 \cdot 00$ & $0 \cdot 91,1 \cdot 10$ \\
\hline Very low food security & 1265 & 12 & $29 \cdot 8$ & $0 \cdot 4$ & $1 \cdot 1$ & $0.4,1.9$ & $40 \cdot 0$ & $2 \cdot 3$ & $1 \cdot 22$ & $1 \cdot 08,1 \cdot 38$ \\
\hline \multicolumn{11}{|l|}{ Asian } \\
\hline High/marginal food security & 1594 & 73 & $22 \cdot 7$ & $0 \cdot 2$ & Ref. & & 4.9 & $1 \cdot 1$ & Ref. & \\
\hline Low food security & 474 & 23 & $24 \cdot 3$ & $0 \cdot 5$ & $1 \cdot 1$ & $0 \cdot 3,2 \cdot 0$ & $10 \cdot 9$ & $3 \cdot 3$ & $1 \cdot 57$ & $0 \cdot 95,2 \cdot 60$ \\
\hline Very low food security & 110 & 4 & $24 \cdot 3$ & $0 \cdot 7$ & $0 \cdot 4$ & $-1 \cdot 2,1 \cdot 9$ & $10 \cdot 6$ & $4 \cdot 5$ & 0.99 & $0.37,2.65$ \\
\hline \multicolumn{11}{|l|}{ Multi-racial } \\
\hline High/marginal food security & 382 & 60 & $26 \cdot 4$ & 0.5 & Ref. & & $17 \cdot 9$ & $3 \cdot 0$ & Ref. & \\
\hline Low food security & 156 & 22 & $26 \cdot 9$ & $1 \cdot 0$ & -0.2 & $-2 \cdot 0,1 \cdot 6$ & $27 \cdot 3$ & $5 \cdot 3$ & $1 \cdot 25$ & $0.80,1.97$ \\
\hline Very low food security & 190 & 18 & $29 \cdot 3$ & $1 \cdot 0$ & $0 \cdot 9$ & $-1 \cdot 1,2 \cdot 9$ & $36 \cdot 7$ & $5 \cdot 2$ & $1 \cdot 37$ & $0.98,1.92$ \\
\hline
\end{tabular}

Ref., reference category.

*From linear and Poisson regression models adjusted for age categories, birthplace, English proficiency, general health status, smoking status, family type, employment status, poverty level and education level. Models for all low-income women additionally adjusted for race/ethnicity. 
birthplace or socio-economic status, as measured by poverty level, education or employment status at the time of survey. Our results are consistent with previous studies demonstrating that low or very low food security is related to higher BMI and higher prevalence of obesity among low-income, California adults; although we found that this association was significantly modified by gender and race/ethnicity.

One previous study had examined the potential modifying effect of race/ethnicity on the association between food insecurity and obesity. In their analysis of the 1998-99 California Women's Health Survey, Adams and colleagues reported that 'non-white' women (Asian, black or Hispanic) with very low food security had nearly threefold higher odds of being obese compared with their food-secure counterparts ${ }^{(9)}$. Possibly due to small sample sizes, associations were not examined within each of the minority racial/ethnic groups. No significant association was reported among white women. Our results are consistent with those of Adams et al., and further suggest that the associations they found may be driven primarily by the large proportion of Hispanic men and women in this subgroup. Two other studies have reported that food insecurity and obesity are positively associated among Hispanics in the USA ${ }^{(7,10)}$. These findings are similar to studies of food security in Latin American countries at advanced stages of the nutrition transition. In Brazil, for example, mild food insecurity was associated with a higher prevalence of obesity in women, and severe food insecurity was strongly associated with obesity in adolescent females ${ }^{(29,30)}$. By contrast, in other Latin American countries like Colombia, that are at earlier stages of the transition, food insecurity has been related to underweight ${ }^{(31)}$.

Among Asian women, low food security but not very low food security was related to higher BMI. Future studies should examine the validity of the food security questions among Asians and examine associations with BMI in a larger sample to better understand the nature of this association. We also did not find significant associations between food insecurity and BMI or obesity among NH whites, African Americans, Asian men or multi-racial women. These findings do not necessarily contradict what was previously known about this association, as most studies have not provided race/ethnicity-stratified estimates. As we showed, very low food security predicted higher BMI and obesity prevalence in the entire CHIS sample, but upon stratification, it became apparent that these associations were driven by low-income Hispanic adults. As individuals may cope with food insecurity in different ways that can affect their weight outcomes, these strategies are not necessarily reflected in a 'one-size-fits-all' approach between food insecurity and obesity. In a review of eight studies examining the food insecurity and obesity association, Dinour and colleagues concluded that the relationship between food insecurity and obesity may 'only exist among women of certain races/ethnicities', although this may be attributed to the fact that many of the studies reviewed were conducted only among women ${ }^{(32)}$.

The mechanisms to explain the association between food insecurity and obesity are complex and combine both individual-level and environmental factors. In a recent review, Franklin and colleagues suggested that gender, marital status and participation in food assistance programmes may modify or mediate the association between food insecurity and obesity in adults ${ }^{(33)}$. Studies examining these associations have found that the effect of food insecurity on weight gain and obesity was generally stronger in women compared with men ${ }^{(13)}$. Food-insecure women who were never married were less likely to be obese than women who were married, partnered or widowed; marital status did not modify the results for men ${ }^{(14)}$. There were mixed results for participation in food assistance programmes as an intermediate in the causal pathway between food insecurity and obesity. As such, the role of food assistance should be further investigated.

Another likely mediator of the association is dietary behaviour, where food-insecure individuals may reduce the quality and/or quantity of foods consumed ${ }^{(4)}$. In a USDA report, it was observed that food-insecure women consumed the same amount of energy as food-secure women, but had significantly worse dietary quality, as measured by the Healthy Eating Index ${ }^{(34)}$. The association between food insecurity and lower dietary quality was also observed in another study of adults residing in the Mississippi Delta ${ }^{(35)}$. In two studies of Latino households with children, household food insecurity was associated with lower household variety of nutrient-dense foods, such as fruits and vegetables, although traditional foods (e.g. beans, white rice, corn tortillas) were not affected $^{(36,37)}$. These studies suggest that food-insecure adults may cope with food insecurity by altering their dietary quality to consume less nutrient-dense foods and more traditional foods. Over time, this may lead to higher total energy intake, weight gain and development of obesity-related complications. In one recent study, it was observed that food insecurity was associated with a threefold higher risk of type 2 diabetes in Latinas ${ }^{(38)}$.

Unlike previous studies that have focused on the association between food insecurity and obesity in women, our results suggest that food-insecure Hispanic and multi-racial men may be as susceptible to obesity as food-insecure Hispanic women. As many food-insecure Hispanic or multi-racial men might reside in the same household as food-insecure women, it is likely that the same mechanisms of household food variety, and cultural dietary behaviours or food preferences, are shared by all food-insecure individuals in the household.

The present study has several strengths. First, the sample size was large and included many persons of minority racial/ ethnic groups; this ensured adequate power to estimate 
gender- and race/ethnicity-specific associations of food insecurity with BMI and obesity. In each survey year, CHIS oversampled African Americans, Hispanics/Latinos, Koreans, Vietnamese and other people from counties with racially/ethnically diverse populations ${ }^{(39)}$. Second, the availability of information on a large number of sociodemographic characteristics allowed us to adjust estimates of association for key potential confounders.

The study is limited by its cross-sectional design. As food insecurity and obesity were measured concurrently, we cannot examine how the duration of food insecurity affects weight change over time. Wilde and Peterman examined the association between food insecurity and obesity in the 1999-2002 National Health and Nutrition Examination Surveys and found that women and men in food-insecure households were more likely to gain weight over a 2-year period than individuals in food-secure households ${ }^{(13)}$. Thus, it is possible that our results represent the effect of food insecurity on weight status. On the other hand, other longitudinal studies suggest that obesity can also affect food security status. Using data from the National Longitudinal Survey of Youth, Cawley observed that race/ethnicity modified the effect of obesity on earned wages, where higher body weight was associated with lower wages for white women and higher wages for African American men ${ }^{(40)}$. In another study of pregnant women, obese women were more likely to be food insecure at 2 years postpartum than non-obese women ${ }^{(41)}$. More longitudinal studies are needed to study how experiences of food insecurity may affect long-term weight status and whether weight status may affect the risk of food insecurity in vulnerable populations. Targeted intervention studies to address food insecurity may also improve the understanding of how different coping strategies may differentially affect dietary behaviours and weight status in specific gender or racial/ethnic groups.

Another limitation of the study is that, although the CHIS sample is meant to be representative of California's diverse adult population, certain adults at the highest levels of food insecurity may have been excluded from the sampling strategy (e.g. adults without landlines/ cellular phones, adults residing in shelters or homeless adults). The overall response rates of the surveys ranged from $18 \cdot 7 \%$ to $33 \cdot 5 \%{ }^{(22-24)}$, which is arguably low, and may have resulted in biased results if adults who responded to the survey were systematically different from adult non-responders. However, CHIS response rates are consistent with the general decline in response rates observed in other random-digit-dial telephone surveys ${ }^{(42)}$. In order for these low response rates to induce bias in our estimates, both food-insecure and obese adults would have to be less willing to participate in CHIS, which we acknowledge as a possibility.

Food security status was measured using the USDA Household Food Security Survey Module: Six-Item Short Form. The main limitation of using the shortened food security questionnaire is that it may not fully capture the more severe experiences of childhood or extreme adult hunger ${ }^{(25)}$. The food security questionnaire was not administered to individuals with household incomes $>200 \%$ of the FPL, which may underestimate the true prevalence of food insecurity in the CHIS sample. However, prevalences of food insecurity in the CHIS sample were very similar to national prevalences estimated by the full eighteen-item Household Food Security Survey Module for the entire US population. Advantages of using the shortened food security questionnaire include less respondent burden and producing estimates of food insecurity with minimal bias ${ }^{(25)}$.

Another limitation is the use of self-reported height and weight data to estimate BMI. Since height is often overreported and weight under-reported, we anticipate that the true BMI of the study respondents may be underestimated, which may have decreased precision and attenuated the observed associations between food insecurity and obesity. As food security status was self-reported, it is possible that individuals who underestimate their BMI may also misreport their food security status and that these errors may be random or systematic depending on the respondent (e.g. less English proficiency, fewer years of education or higher poverty level). Although the USDA Food Security Survey Module has been validated in a Latino/Hispanic population $^{(43)}$, correlated errors may have exaggerated the true association between food insecurity and obesity. Lastly, as California has its own unique culture, the findings may also not be generalizable to persons residing in other states and future studies may wish to confirm these associations in other statewide or national health studies.

Despite these limitations, our study suggests that food insecurity is associated with BMI and obesity predominantly in low-income, Hispanic men and women, Asian women and multi-racial men. While other California adults also experience food insecurity and suffer from obesity, there was no evidence of a positive relationship in $\mathrm{NH}$ whites, African Americans, Asian men or multi-racial women. In order to infer a causal relationship, longitudinal studies are needed to determine when individuals are most vulnerable to the effects of food insecurity on long-term weight and health status. The associations between food insecurity and obesity among Hispanics and other racial/ethnic groups should be further investigated in order to examine the mechanisms in which these associations exist and to help design targeted interventions to alleviate food insecurity and improve health outcomes in these groups.

\section{Acknowledgements}

This publication was supported by a National Institutes of Health Grant 5 T32 CA009001-35. The authors have no conflicts of interest to declare. C.W.L., D.R.W. and E.V. 
designed the research; C.W.L conducted the research and performed statistical analysis; C.W.L., D.R.W. and E.V. wrote the paper, and C.W.L. had primary responsibility for the final content. All authors have read and approved the final manuscript.

\section{References}

1. Wang Y \& Beydoun MA (2007) The obesity epidemic in the United States - gender, age, socioeconomic, racial/ethnic, and geographic characteristics: a systematic review and meta-regression analysis. Epidemiol Rev 29, 6-28.

2. Flegal KM, Carroll MD, Ogden CL et al. (2010) Prevalence and trends in obesity among US adults, 1999-2008. JAMA 303, 235-241.

3. Nord M \& Carlson S (2008) Household Food Security in the United States, 2007. Economic Research Report no. ERR-66. Washington, DC: US Department of Agriculture, Economic Research Service.

4. Nord M, Coleman-Jensen A, Andrews M et al. (2010) Housebold Food Security in the United States, 2009. Economic Research Report no. ERR-108. Washington, DC: US Department of Agriculture, Economic Research Service.

5. Nord M \& Coleman-Jensen A (2011) Food Security in the United States: Key Statistics and Graphics. Washington, DC: US Department of Agriculture, Economic Research Service.

6. Vozoris NT \& Tarasuk VS (2003) Household food insufficiency is associated with poorer health. J Nutr 133, $120-126$.

7. Bhattacharya J, Currie J \& Haider S (2004) Poverty, food insecurity, and nutritional outcomes in children and adults. $J$ Health Econ 23, 839-862.

8. Olson CM (1999) Nutrition and health outcomes associated with food insecurity and hunger. J Nutr 129, 2S Suppl., 521S-524S.

9. Adams EJ, Grummer-Strawn L \& Chavez G (2003) Food insecurity is associated with increased risk of obesity in California women. J Nutr 133, 1070-1074.

10. Kaiser LL, Townsend MS, Melgar-Quinonez HR et al. (2004) Choice of instrument influences relations between food insecurity and obesity in Latino women. Am J Clin Nutr 80, 1372-1378.

11. Townsend MS, Peerson J, Love B et al. (2001) Food insecurity is positively related to overweight in women. J Nutr 131, 1738-1745.

12. Martin KS \& Ferris AM (2007) Food insecurity and gender are risk factors for obesity. J Nutr Educ Behav 39, 31-36.

13. Wilde PE \& Peterman JN (2006) Individual weight change is associated with household food security status. $J$ Nutr 136, 1395-1400

14. Hanson KL, Sobal J \& Frongillo EA (2007) Gender and marital status clarify associations between food insecurity and body weight. $J$ Nutr 137, 1460-1465.

15. Parker ED, Widome R, Nettleton JA et al. (2010) Food security and metabolic syndrome in US adults and adolescents: findings from the National Health and Nutrition Examination Survey, 1999-2006. Ann Epidemiol 20, 364-370.

16. UCLA Center for Health Policy Research (2008) California Health Interview Survey. http://www.chis.ucla.edu/ (accessed April 2011)

17. US Census Bureau (2012) California QuickFacts from the US Census Bureau. http://quickfacts.census.gov/qfd/states/ 06000.html (accessed April 2011).

18. UCLA Center for Health Policy Research (2008) Where does CHIS interview? http://www.chis.ucla.edu/where-chis.html (accessed April 2011).
19. Seligman HK, Laraia BA \& Kushel MB (2010) Food insecurity is associated with chronic disease among lowincome NHANES participants. J Nutr 140, 304-310.

20. Nord M (2007) Characteristics of Low-Income Households with Very Low Food Security. Economic Information Bulletin no. EIB-25. Washington, DC: US Department of Agriculture, Economic Research Service.

21. 2009 Federal Poverty Guidelines. US Department of Health and Human Services.

22. California Health Interview Survey (2009) CHIS 2007 Methodology Series: Report 4-Response Rates. Los Angeles, CA: UCLA Center for Health Policy Research.

23. California Health Interview Survey (2007) CHIS 2005 Methodology Series: Report 4-Response Rates. Los Angeles, CA: UCLA Center for Health Policy Research.

24. California Health Interview Survey (2005) CHIS 2003 Methodology Series: Report 4 - Response Rates. Los Angeles, CA: UCLA Center for Health Policy Research.

25. Bickel G, Nord M, Price C et al. (2000) Guide to Measuring Housebold Food Security. Alexandria, VA: US Department of Agriculture, Food and Nutrition Service, Office of Analysis, Nutrition and Evaluation.

26. Coleman-Jensen A, Nord M, Andrews M et al. (2011) Food Security in the United States in 2010. Economic Research Report no. ERR-125. Washington, DC: US Department of Agriculture, Economic Research Service.

27. Nord M, Andrews M \& Carlson S (2006) Household Food Security in the United States, 2005. Economic Research Report no. ERR-29. Washington, DC: US Department of Agriculture, Economic Research Service.

28. Nord M, Andrews M \& Carlson S (2004) Household Food Security in the United States, 2003. Economic Research Report no. ERR-42. Washington, DC: US Department of Agriculture, Economic Research Service.

29. Kac G, Velasquez-Melendez G, Schlussel MM et al. (2012) Severe food insecurity is associated with obesity among Brazilian adolescent females. Public Health Nutr (Epublication ahead of print version).

30. Velasquez-Melendez G, Schlussel MM, Brito AS et al. (2011) Mild but not light or severe food insecurity is associated with obesity among Brazilian women. J Nutr 141, 898-902.

31. Isanaka S, Mora-Plazas M, Lopez-Arana S et al. (2007) Food insecurity is highly prevalent and predicts underweight but not overweight in adults and school children from Bogota, Colombia. J Nutr 137, 2747-2755.

32. Dinour LM, Bergen D \& Yeh MC (2007) The food insecurity-obesity paradox: a review of the literature and the role food stamps may play. J Am Diet Assoc 107, 1952-1961.

33. Franklin B, Jones A, Love D et al. (2011) Exploring mediators of food insecurity and obesity: a review of recent literature. J Community Health 37, 253-264.

34. Basiotis PP \& Lino M (2002) Food Insufficiency and Prevalence of Overweight Among Adult Women. Alexandria, VA: US Department of Agriculture, Center for Nutrition Policy and Promotion.

35. Champagne CM, Casey PH, Connell CL et al. (2007) Poverty and food intake in rural America: diet quality is lower in food insecure adults in the Mississippi Delta. J Am Diet Assoc 107, 1886-1894.

36. Kaiser LL, Melgar-Quinonez H, Townsend MS et al. (2003) Food insecurity and food supplies in Latino households with young children. J Nutr Educ Behav 35, 148-153.

37. Matheson DM, Varady J, Varady A et al. (2002) Household food security and nutritional status of Hispanic children in the fifth grade. Am J Clin Nutr 76, 210-217.

38. Fitzgerald N, Hromi-Fiedler A, Segura-Perez S et al. (2011) Food insecurity is related to increased risk of type 2 diabetes among Latinas. Ethn Dis 21, 328-334. 
39. UCLA Center for Health Policy Research (2008) Sample Design Description for CHIS 2003 (Original Plan). http:// www.chis.ucla.edu/pdf/sample_desc_2003.pdf （accessed April 2011).

40. Cawley J (2004) The impact of obesity on wages. J Hum Resour 39, 451-474.

41. Olson CM \& Strawderman MS (2008) The relationship between food insecurity and obesity in rural childbearing women. J Rural Health 24, 60-66.
42. Smith DW (2006) Sources of variation in response rates to the Behavioral Risk Factor Surveillance System. ASA Section on Survey Research Methods 3721-3726; available at http:// www.amstat.org/sections/srms/proceedings/y2006/Files/ JSM2006-000708.pdf

43. Kaiser L \& Melgar-Quiñonez H (2001) RIDGE Project Summary - Validation of Food Security Instruments in Hispanic Households. Washington, DC: US Department of Agriculture, Economic Research Service. 